\title{
Transmit Power Minimization using Fuzzy Rule based System in Relay Assisted Cognitive Radio Networks
}

\author{
Kiran Sultan \\ Dept. of CIT \\ King Abdul Aziz University \\ Jeddah, Saudi Arabia
}

\author{
Bassam A. Zafar \\ Dept. of CIT \\ King Abdul Aziz University \\ Jeddah, Saudi Arabia
}

\author{
Babar Sultan \\ Dept. of Telecomm. Engg. \\ MCS, NUST \\ Rawalpindi, Pakistan
}

\begin{abstract}
Transmit power minimization is one of the major research challenges in Relay-Assisted Cognitive Radio Networks. In this process, the transmit power of each individual relay is adjusted in such a way that the overall transmit power consumption at the relay network is minimized while satisfying the minimum Quality-of-Service (QoS) requirements of primary and secondary networks. In this paper, a similar constrained optimization problem is focused in which a secondary source-destination pair is assisted by a potential relay network having Cognitive Radio capabilities. A Fuzzy Rule Based System (FRBS) is proposed for intelligent relay selection such that total transmit power at the relay network is minimized while achieving the desired signal-to-noise ratio (SNR) at the destination and keeping the primary communication undisturbed. The effectiveness of the proposed scheme is highlighted through simulation results.
\end{abstract}

\section{Keywords}

Cognitve Radio Network, Underlay Spectrum Sharing, Cooperative Communication, Amplify-and-Forward, Fuzzy Rule Based System

\section{INTRODUCTION}

Cognitive Radio (CR), coined by Joseph Mitola, alleviates the spectrum underutilization problem by enabling the secondary users (SUs) to coexist with the primary users (PUs) under different spectrum sharing modes [1]. In an underlay mode of operation [2], the transmit power of the SUs are much constrained in order to keep the interference offered to the PUs below the predefined threshold. Optimal transmit power allocation in such energy-constrained environment is one of the major research challenges in Cognitive Radio Networks (CRNs). The situation becomes more complicated, when large physical separation is involved between the secondary sourcedestination pair making direct communication impossible. Cooperative communication has gained a lot of attention in the wireless adhoc networks due to its capabilities to combat channel fading and to enhance the overall system performance by coverage area extension, power saving and increasing capacity [3]. In cooperative communication, an intermediate node between source-destination pair acts as a relay to forward the received message [4]. Amplify-and-Forward (Non-Regenerative Relaying) and Decode-and-Forward (Regenerative Relaying) are two classic and most commonly employed relaying protocols [5]. Thus, inspired by CR and cooperative communication techniques, Relay assisted Cognitive Radio Network (RCRN) has emerged as one of the candidate solutions to face the highlighted challenges of the underlay networks [6]. However, to engage all the relays between the source-destination pair might be risky in terms of total interference offered by the relay network to the active PU [7]. Fortunately, a fascinating solution to this problem exists in RCRNs in the form of relay selection. Few research contributions for the performance enhancement of secondary communication while guaranteeing to satisfy the interference constraints of the PUs in RCRNs are highlighted as follows. In [8], authors considered RCRN and proposed performance enhancement scheme through optimal transmit power allocation in such a way that the interference threshold constraint of the coexisting primary network was satisfied. Multiple relay selection schemes for underlay CRN were proposed by [9], aiming to maximize the SNR at the destination while keeping the interference offered by the source and the selected relay network below the preset interference threshold. In [10], authors considered a sourcedestination pair assisted by a cognitive relay for communication due to the absence of line-of-sight path and solved the sum transmit power minimization problem at the secondary network while satisfying the Signal to Interference plus Noise Ratio (SINR) constraint, interference constraint and individual transmitter's power constraint.

Fuzzy Logic has recently gained tremendous attention in wireless communication research especially in adaptive communications. Atta-ur-Rahman et al. [11] proposed a fuzzy rule based system (FRBS) for adaptive coding, modulation and power in orthogonal frequency division multiplexing (OFDM) based Hyperlans such that the overall throughput of the system was maximized while transmit power and bit error rate (BER) constraints were satisfied. It was shown through the simulations that the proposed scheme outperformed the famous Water-filling algorithm for multicarrier loading. Applications of Fuzzy Logic (FL) have already been found in CRNs [12]-[14]. Some methods of FL based transmit power allocation and power management in CRNs are highlighted as under. A power control mechanism based on FL for spectrum sharing in CRNs was proposed in [15]. In [16], Tabakovic et al. applied FL to design a transmit power controller which enables the secondary communication with the desired QoS requirements, while ensuring that the interference offered to the PU is minimized. In [17], authors proposed a power control method based on FL for the coexisting primary and secondary networks, aiming to maximize the spectrum usage efficiency while ensuring the desired QoS requirements for the primary network. However, the application of FL for transmit power minimization at the cognitive relay network, while ensuring to satisfy the minimum QoS requirement of the SU and interference threshold of the PU in an underlay spectrum sharing model has not been done so far to the best of our knowledge.

In this paper, a novel idea of employing FRBS for multiple relay selection is proposed. The proposed FRBS selects the optimal relay subsets in such a way that the overall power consumed in the relay network is minimized while satisfying target SNR threshold and interference threshold. By taking 
into account the power received at each relay, channel coefficients of relay-destination and relay-PU links; FRBS assigns a Relay Selection Factor (RSF) to each candidate relay in the network to make a decision about selection of that particular relay.

The remaining paper is organized as follows. In section II, we provide details of the system model and formulate the multiple relay selection problem. The proposed relay selection scheme based on FL is explained in section-III. Section-IV explains FRBS designed to solve the highlighted problem. Section V illustrates the simulation results for the performance evaluation, while the conclusion about the whole work is made in section-VI.

\section{SYSTEM MODEL AND PROBLEM FORMULATION}

We consider a dual-hop RCRN as shown in Figure 1 comprising a single source-destination pair assisted by $J$ relays having CR capabilities. It is assumed that the line-ofsight (LOS) path is absent between the source destination pair due to large physical separation, thus the end-to-end communication relies on the intermediate relays. The entire relay network shares the licensed spectrum with the PU, therefore, it is ensured that the total interference offered by the relay network does not violate the predefined interference threshold. Let $\left\{g_{S R_{j}}\right\}_{j=1}^{J},\left\{g_{R_{j} D}\right\}_{j=1}^{J}$ and $\quad\left\{g_{R_{j} Q}\right\}_{j=1}^{J}$ denote the independent and identically distributed sourcerelay, relay-destination and relay-PU channel coefficients of the relay network.

In this Rayleigh flat fading scenario, it is assumed that the perfect channel state information (CSI) is available at each $j^{\text {th }}$ relay, and the FRBS assisted relay selection and transmit power control scheme relies on this information make a decision.

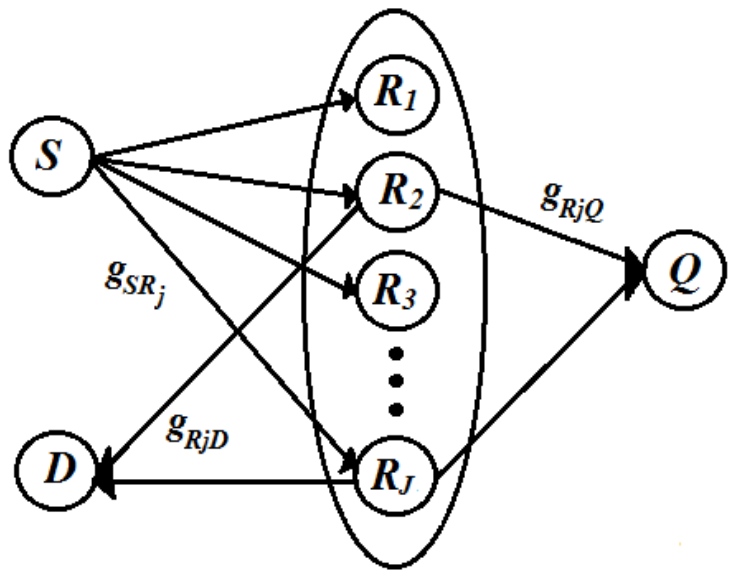

Fig 1: The System Model

Amplify-and-Forward (AF) protocol is employed at each relay, thus, each selected relay just amplifies the received message and forwards it to the destination. The transmission process begins at the source $S$ and is completed in two phases. In the first phase, each relay listens to the signal broadcast by the source. In the second phase, FRBS assisted relay selection scheme picks the optimal combination of relays to amplify and forward the received message. Relay selection aims to choose the best subset of the potential relay set that is capable of minimizing the sum transmit power at the relay network while satisfying the minimum QoS requirements. Let $P_{j}$ represents the transmit power of the $j^{\text {th }}$ relay defined according to $\mathrm{AF}$ relaying as,

$$
P_{j}=A_{j}^{2}\left(P_{S}\left|g_{S R_{j}}\right|^{2}+N_{0}\right)
$$

where, $P_{S}$ represents the transmit power of the source, and $N_{0}$ is the additive white Gaussian noise (AWGN) received at the $j^{\text {th }}$ relay.

The SNR received at the destination due to $j^{\text {th }}$ relay link is given by [18],

$$
\gamma_{j}=\frac{\gamma_{S R_{j}} \gamma_{R_{j} D}}{1+\gamma_{S R_{j}}+\gamma_{R_{j} D}}
$$

where,

$\gamma_{S R_{j}}=\frac{P_{S}\left|g_{S R_{j}}\right|^{2}}{N_{0}}$ and $\gamma_{R_{j} D}=\frac{P_{j}\left|g_{R_{j} D}\right|^{2}}{N_{0}}$

denote the SNR of the $j^{\text {th }}$ source-relay link and relaydestination link respectively.

Thus, the total end-to-end SNR due to the potential relay network is given as,

$$
\gamma_{D}=\sum_{j=1}^{J} \gamma_{j}=\sum_{j=1}^{J} \frac{\gamma_{S R_{j}} \gamma_{R_{j} D}}{1+\gamma_{S R_{j}}+\gamma_{R_{j} D}}
$$

The key requirement of the underlay RCRNs is to satisfy the interference threshold all the time. The interference constraint imposed on the potential relay network is expressed as,

$$
I=\sum_{j=1}^{J} I_{j}=\sum_{j=1}^{J} P_{j}\left|g_{R_{j} Q}\right|^{2} \leq \beta
$$

where $\beta$ denotes the interference threshold.

The above interference constraint enforces the relay network to transmit at low power, decreasing the SNR achieved at the relay-destination links. Thus, in order to satisfy $\beta$, it might not always be possible to enable the secondary transmissions using all potential relays, rather relay selection needs to be performed to select a subset $\Omega_{S}$ of a potential relay set that intelligently enables the secondary communication, while satisfying the constraints. Thus, Eq. (3) takes the form under multiple relay selection problem as,

$$
\gamma_{D}=\sum_{j \in \Omega_{s}} \gamma_{j}=\sum_{j \in \Omega_{s}} \frac{\gamma_{S R_{j}} \gamma_{R_{j} D}}{1+\gamma_{S R_{j}}+\gamma_{R_{j} D}}
$$

where $\Omega_{s}$ denotes the subset of relays selected to forward the received message after amplification. 
In literature, tight upper and lower bounds for $\gamma_{j}$ exist for a comprehensive performance analysis [19]. In terms of these bounds, $\gamma_{j}$ is expressed as, $\gamma_{j}^{l b} \leq \gamma_{j} \leq \gamma_{j}^{u b}$, where, $\gamma_{j}^{l b} \leq \frac{1}{2} \min \left(\gamma_{S R_{j}}, \gamma_{R_{j} D}\right)$ and $\gamma_{j}^{u b} \leq \min \left(\gamma_{S R_{j}}, \gamma_{R_{j} D}\right)$. The bounds clearly show that $\gamma_{j}$ solely depends on $\gamma_{S R_{j}}$ when $\gamma_{R_{j} D}>\gamma_{S R_{j}}$. However, $\gamma_{j}$ can be enhanced for the case when $\gamma_{R_{j} D}<\gamma_{S R_{j}}$ to approach the upper bound. Thus, any attempt to improve $\gamma_{j}$ for those relays which exhibit $\gamma_{S R_{j}}<\gamma_{R_{j} D}$ will not result in any significant enhancement as $\gamma_{j}<\gamma_{S R_{j}}$ in this case. This interesting behavior of $\gamma_{j}$ makes the problem of transmit power minimization through relay selection more tractable. The proposed FRBS assisted relay selection and power allocation scheme serves the purpose and attempts to choose the best combination of relays in which each selected relay is able to increase the corresponding $\gamma_{R_{j} D}$ by performing minimum amplification, such that the total SNR achieved at the relay-destination links must satisfy predefined SNR threshold $\eta$. The proposed FRBS will be explained in the next section.

Thus, the mathematical formulation of the relay subset selection problem is given as,

$$
\begin{gathered}
\min \left(\sum_{j^{\prime} \in \Omega_{S}} P_{j^{\prime}}\right) \\
\text { s.t. } \\
\gamma_{2}=\sum_{j^{\prime} \in \Omega_{S}} P_{j^{\prime}}\left|g_{R_{j^{\prime}} D}\right|^{2} \geq \eta \\
I=\sum_{j^{\prime} \in \Omega_{S}} P_{j^{\prime}}\left|g_{R_{j^{\prime}} Q}\right|^{2} \leq \beta
\end{gathered}
$$

where, the cardinality of the selected subset $\Omega_{S}$ is $J^{\prime} \leq J$.

\section{THE PROPOSED ALGORITHM}

FRBS assisted relay selection scheme works as follows. The inputs to the FRBS are the transmit power $P_{j}$ for each $j^{\text {th }}$ relay, and the ratio of coefficients $g_{R_{j} D}$ and $g_{R_{j} Q}$, and RSF is assigned to each $j^{\text {th }}$ candidate relay. Let $\bar{R}=\left[R S F_{1}, R S F_{2}, \cdots \cdot \cdot R S F_{J}\right]$ be the RSF vector of the potential relay network obtained from FRBS. The relays are then picked up in the descending order of the RSF in $\bar{R}$. The flowchart of the proposed technique is shown in Figure 2.

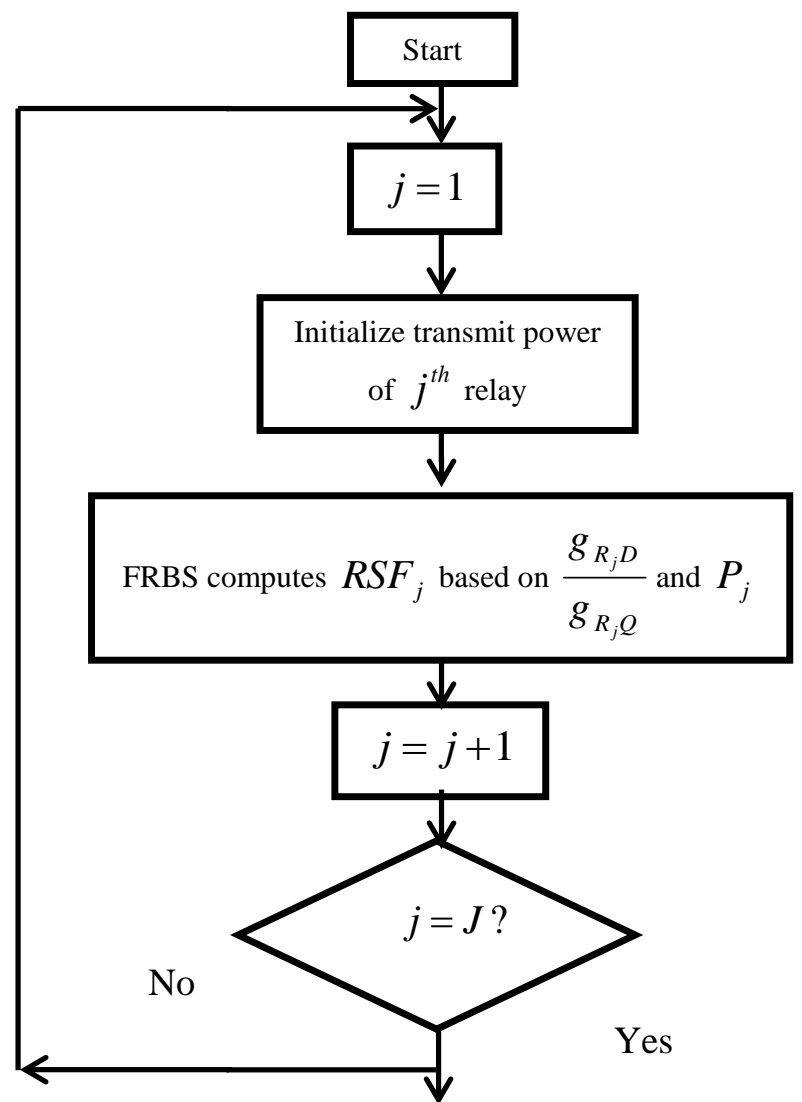

Pick the relays in the descending order of RSF and perform transmit power tuning of each selected relay

$$
\begin{gathered}
\text { Outputs: } \\
\Omega_{s}, \sum_{j \in \Omega_{s}} \gamma_{R_{j} D}
\end{gathered}
$$

Fig 2: Flow Chart of the proposed FRBS assisted Relay Selection System Design

\section{FUZZY RULE BASED SYSTEM}

FRBS is employed to perform relay selection as explained earlier and is designed in MATLAB standard FL toolbox. The proposed Fuzzy Logic System (FLS) is shown in Figure 3.

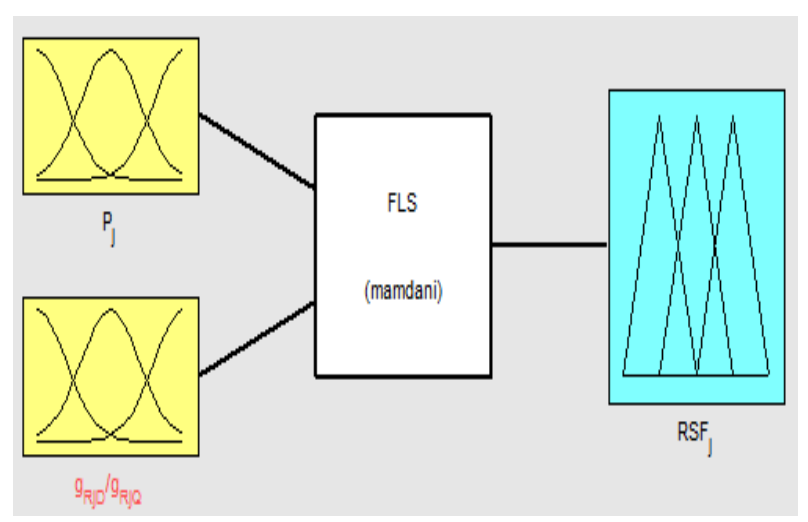

Fig 3: Proposed FRBS 
As Figure 3 shows, the power $P_{j}$ transmitted by the $j^{\text {th }}$ relay in the relay network and the ratio of relay-destination channel coefficient relative to the relay-PU channel coefficient act as the antecedents and the fuzzy inference engine computes a RSF for each candidate relay based on the fuzzy rules.
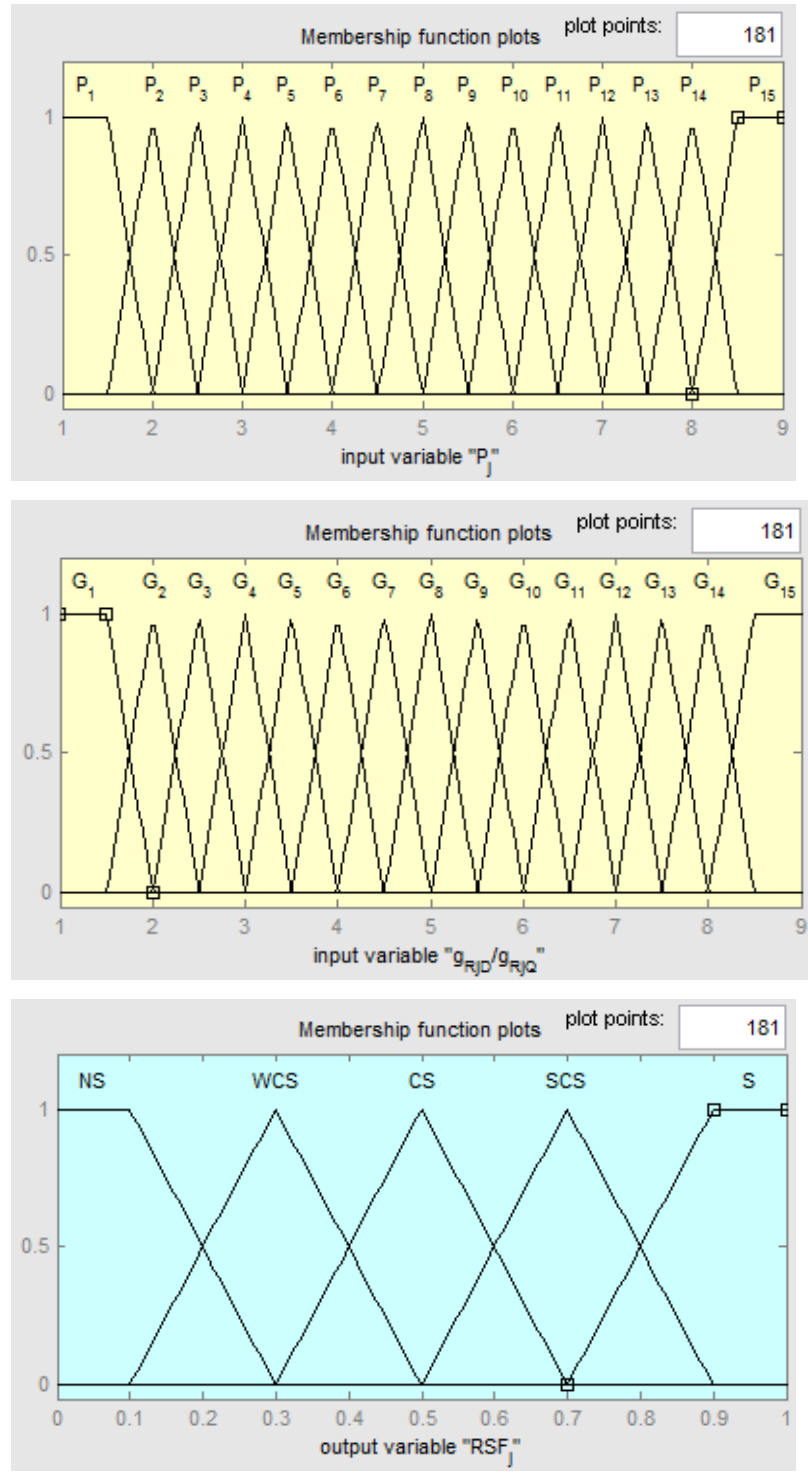

Fig 4: Fuzzy Sets for the antecedents and the consequents

Referring to Figure 4, to make the whole design sophisticated and accurate, the whole range of the inputs and the output are covered with sufficient number of fuzzy sets. Fifteen fuzzy sets are used for the both antecedents $P_{j}$ and $\frac{g_{R_{j} D}}{g_{R_{j} Q}}$ and five fuzzy sets for the consequent $R S F_{j}$, with trapezoidal MFs for the minimum and maximum levels and triangular MFs for the intermediate levels. The fuzzy sets for the RSF are: Not Selected (NS), Weak Consideration for Selection (WCS), Consider for Selection (CS), Strong Consideration for Selection (SCS) and Selected (S). There are $15 \times 15=225$ "IF-THEN" rules with each $m^{\text {th }}$ rule of the form,

$$
\begin{gathered}
R^{m}: \text { IF } P_{1}^{m} \text { is } P_{13}, \frac{g_{R_{j} D}^{m}}{g_{R_{j} Q}^{m}} \text { is } G_{15}, \text { THEN, } \text { RSF }_{1}^{m} \text { is } S \\
m=1,2, \cdots \cdots, M
\end{gathered}
$$

where, $M$ represents the total number of rules.

There are three steps involved in FLS decision procedures, namely fuzzification, fuzzy inference engine (FIE) and defuzzification [20]. The fuzzification process fuzzifies each input linguistic variable to produce a set of fuzzy numbers. This is done by comparing each input variable with the predefined MFs to allocate the variable, a value between 0 and 1. Standard triangular fuzzifier is employed for this purpose, with AND as min and OR as MAX. FIE, containing a predefined rule base, is fed by the fuzzy numbers from the fuzzifier to illustrate the relations between the input and the output variables through IF-THEN rules based on fuzzy reasoning. The rule base contains rules for every possible combination of the input space. Standard Mamdani Inference Engine (MIE) serves the purpose in this work. The fuzzy variables produced by FIE are converted into the crisp values by the Defuzzifier to represent the actual output of the system. Center Average Defuzzifier (CAD) is preferred because of its computationally simplicity.

\section{SIMULATION RESULTS AND DISCUSSIONS}

In this section, we present the simulation results to confirm the effectiveness of FRBS to select the best combination of relays for optimizing system performance. Figure 5 illustrates the rule surface for the proposed FLS. Table 1 provides the parameter sittings for all simulations.

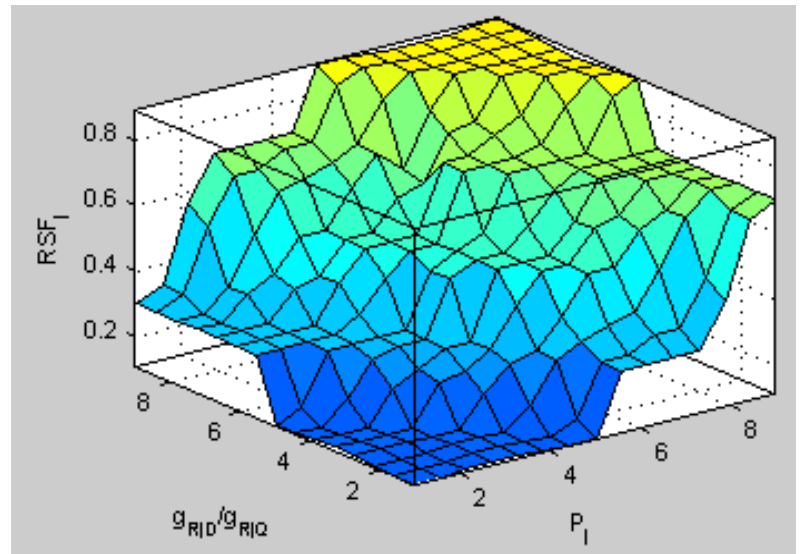

Fig 5: The Rule Surface

Table 1. Parameter Settings

\begin{tabular}{|c|c|}
\hline Parameters & Values \\
\hline$g_{S_{R_{j}}}$ & $0.3-0.9$ \\
\hline$g_{R_{j} Q}$ & $0.1-0.3$ \\
\hline$g_{R_{j} D}$ & $0.4-0.9$ \\
\hline$N_{0}$ & 1 \\
\hline
\end{tabular}


Figure 6 illustrates the behavior of the proposed scheme for different levels of $\beta . \eta$ is set to $1 . P_{S}$ is set to 10 . We consider three different sizes of the potential relay network, i.e. $J=\{5,10,15\}$. As Figure 6(a) shows, overall transmit power of the relay network increases by relaxing $\beta$, because high levels of $\beta$ enable the relays to freely transmit at high power to meet the QoS requirements of the secondary network without interfering the primary signals. However, there is another strong observation that as the size of the potential relay network grows, the total transmit power decreases. This is due to the reason that a large relay network provides more choices to FRBS to perform intelligent relay selection. Figure 6(b) shows the total number of selected relays corresponding to different cases of Figure 6(a). As the figure shows, full cooperative diversity is observed for the interference threshold level $\beta \geq 10 d B$.On the other hand, setting the interference threshold too low makes the relay selection problem very difficult and a single best relay is selected to participate in the communication. Thus, relay selection will become impossible below this level of interference threshold.

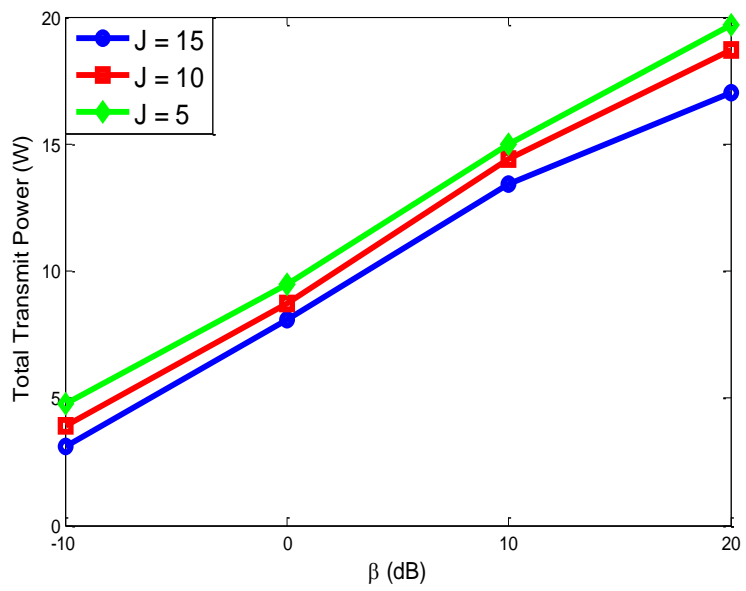

Fig 6(a)

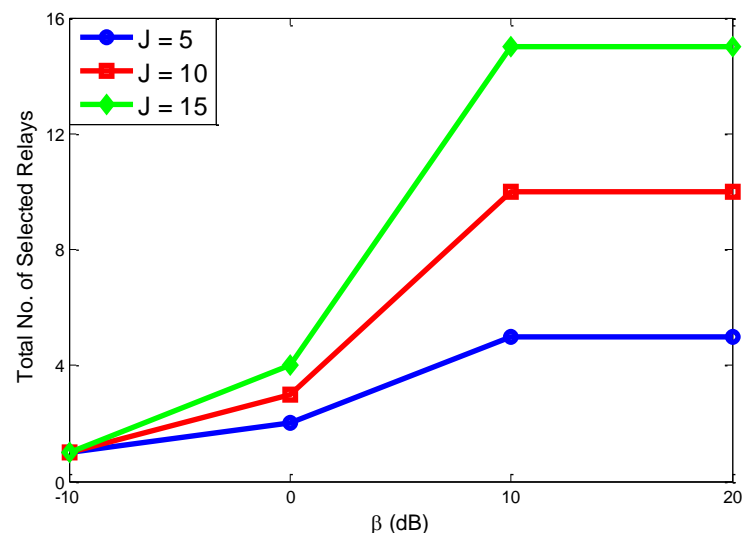

Fig 6(b)

Fig 6: (a) Total Transmit Power vs $\beta$ for $\eta=1$, (b)

Corresponding Total Number of Selected Relays

In Figure 7, the proposed scheme is analyzed for different levels of $\eta$ while $\beta$ is set to $0 d B . P_{S}$ is kept the same as in the case of Figure 6. Again the performance is evaluated considering three different sizes of potential relay networks, i.e. $J=\{5,10,15\}$. As the figure shows, the transmit power of the relay network increases when $\eta$ is relaxed, because in order to satisfy high $\eta$, high transmit power is required at the intermediate relay network. Furthermore, only slight variations in the $\eta$ were possible in order to study the system response, owing to the fact that underlay environments does not allow the SUs to transmit at high power keeping in view the privilege of the PUs. Thus, setting too high $\eta$ eventually makes the secondary communication impossible. Finally, for large potential relay networks, total transmit power significantly decreases, because a large network provides more opportunities to the FRBS to intelligently pick the best combination of relays to satisfy the QoS requirements of both primary and secondary networks while saving the resources available at each node.

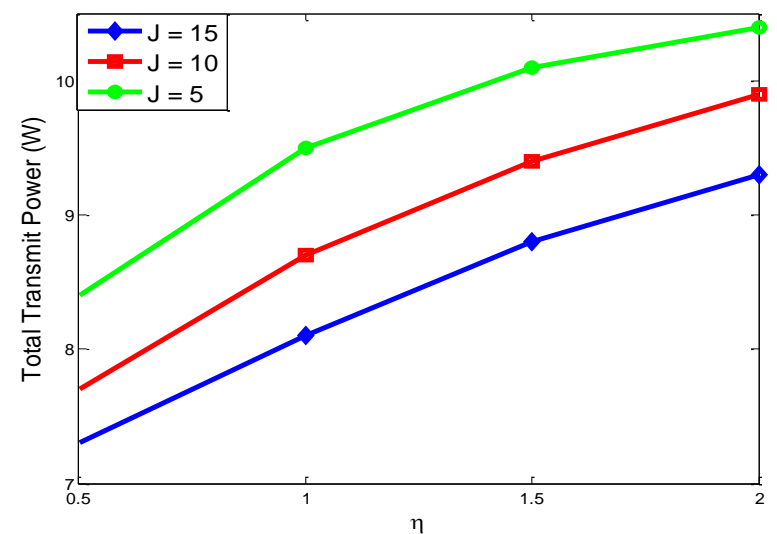

Fig 7: Total Transmit Power vs $\eta$

Figure 8 extends the proposed FRBS assisted relay selection system design to study the behavior of the relay network for different cases of source transmit power. $\beta$ is set to $10 \mathrm{~dB}$. As observed from the figure, the total power transmitted by the relay network significantly decreases by increasing the transmit power of the source because the higher is the power received at the intermediate relay, the lower is the amplification required at each selected relay to satisfy the SNR threshold.

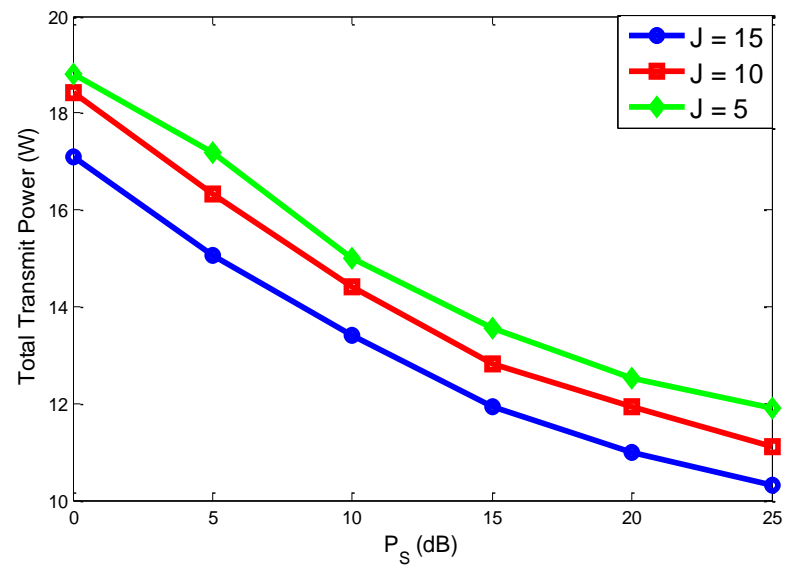

Fig 8: Total Transmit Power of Relay Network for different levels of source transmit power keeping $\beta=10 d B$ and $\eta=1$ 


\section{CONCLUSION}

In this work, Fuzzy Rule Based System (FRBS) is proposed to solve multiple relay selection problem for underlay relay assisted cognitive radio networks. Proposed FRBS takes the channel state information (CSI) of each candidate relay in the potential relay network as an input and assigns a Relay Selection Factor (RSF) to each relay. RSF eventually sets the precedence in which the relays are selected, aiming to enable the coexistence of the primary and the secondary networks with minimum Quality of Service (QoS) requirements, while utilizing the minimum transmit power at the relay network. The simulation results confirm the effectiveness of FRB assisted relay assignment scheme.

\section{REFERENCES}

[1] Shahrasbi, B. and Rahnavard, N. 2011, Rateless-coding based cooperative cognitive radio networks: Design and analysis, IEEE SECON, 224-232.

[2] Lee, J., Wang, H., Andrews, J.G. 2011, Outage Probability of Cognitive Relay Networks with Interference Constraints, IEEE Transactions on Wireless Comm., 390-395.

[3] Chen, D., Ji, H., Li, X. 2011. Optimal Distributed Relay Selection in Underlay Cognitive Radio Networks: An Energy-Efficient Design Approach, IEEE WCNC, 12031207.

[4] Liu, F., Zhang, X., Chen, Z., Wang, Y., Yang, D. 2009. Performance Measure Analysis of Amplify-and-Forward Relaying over Non-identical Nakagami-m Fading Channel, IEEE ICC, 1-6.

[5] Wu, M., Wubben, D., Dekorsy, A. 2011. BER-based Power Allocation for Amplify-and-Forward and Decodeand-Forward Relaying Systems, Int. ITG workshop on Smart Antennas, 1-8.

[6] Sultan, K., Qureshi, I.M., Zubair, M. 2012. SNR Maximization through Relay Selection and Power Allocation for Non-Regenerative Cognitive Radio Networks, IEEE INMIC, 361-364.

[7] Naeem, M., Lee, D.C., Pareek, U. 2010. An Efficient Multiple Relay Selection Sceheme for Cognitive Radio Systems, IEEE ICC, 1-5.

[8] Mietzner, J., Lampe, L., Schober, R. 2009. Distributed Transmit Power Allocation for Multihop Cognitive-
Radio Systems, IEEE Transactions on Wireless Comm., 5187-5201.

[9] Xu, J., Zhang, H., Yuan, D., Jin, Q., Wang C.X. 2011. Novel Multiple Relay Selection Schemes in Two-Hop Cognitive Relay Networks, IEEE CMC, 307-310.

[10] Li, D., Dai, X. 2009. Power Control in Cooperative Cognitive Radio Networks by Geometric Programming, IEEE APCC, 118-121.

[11] Atta-ur-Rahman, Qureshi, I.M., Muzaffar, M.Z. Naseem, M.T. 2012. Adaptive Resource Allocation for OFDM Systems using Fuzzy Rule Base System and Water-filling Principle. SoCPaR, 811-816.

[12] Le, H.T., Ly, H.D. 2008. Opportunistic Spectrum Access Using Fuzzy Logic for Cognitive Radio Networks, IEEE ICCE, 240-245.

[13] Liu, W., Lv, T., Gao, Wang L.W., Liu, B. 2009. A Novel Cooperative Spectrum Sensing Scheme Based on Fuzzy Integral Theory in Cognitive Radio Networks, IEEE WiCom, 1-4.

[14] Ahmed, K., Bashir, F., Najum-ul-Hassan, Ehsan ul Haq M. 2010. Comparative study of centralized cooperative spectrum sensing in cognitive radio networks, IEEE ICSPS, 246-249.

[15] Dey, A., Biswas, S., Panda, S. 2011. A New Fuzzy Rule Based Power Management Scheme for Spectrum Sharing in Cognitive Radio, IEEE ICCIA, 1-4.

[16] Tabakovic, Z., Grgic, S., Grgic, M. 2009. Fuzzy Logic Power Control in Cognitive Radio, IEEE IWSSIP, 1-5.

[17] Mustafa, W., Rakus-Andersson, E. 2010. Fuzzy-based Opportunistic Power Control Strategy in Cognitive Radio Networks, IEEE ISABEL, 1-5.

[18] Hui, H., Zhu, S., Lv, G. 2010. Relay Selection for Lifetime Extension in Amplify-and-Forward Cooperative Networks, IEEE ICC, 1-5.

[19] Amarasuriya, G., Ardakani, M., Tellambura, C. 2010 , Output-Threshold Multiple-Relay-Selection Scheme for Cooperative Wireless Networks. IEEE Transactions on Vehicular Technology. Vol. 59(6), 3091-3097.

[20] Yang, S. and Wu, J. 2010. A Spectrum Sharing Method based on Fuzzy Logic in IEEE 802.22 WRAN, IEEE WCSP, $1-5$. 